\title{
DESARROLLO BIOMÉTRICO EN Aotus nancymae NACIDOS EN CAUTIVERIO
}

\author{
Jéssica Gálvez-Durand B., ${ }^{1}$, Enrique Montoya G. ${ }^{2}$, Hugo Gálvez C. ${ }^{2}$ \\ y Carlos Ique G. ${ }^{2}$
}

\section{Alstract}

A biometrics study of 30 owl monkeys, Aotus nancymae, was conducted at the IVITA Research station in Iquitos, Perú, to determine normal growth parameters during the first six months of life for animals born in captivity. Weight, body, arm, leg and tail length, and cranial circumference measurements were recorded at two week intervals from birth to 26 weeks of age. These measurements were found to correlate exactly with the age of the animals, and application of the multiple linear regression formula $\mathrm{Y}=\mathrm{a}+$ $b_{1} x_{1}+b_{2} x_{2}+b_{3} x_{3}+b_{4} x_{4}+b_{5} x_{5}+b_{6} x_{6}$ accurately predicts the age of captive Aotus from birth to one year.

Key words: Biometrics measurements, Aotus, captivity, growth parameters.

\section{Resumen}

Un estudio biométrico en Aotus nancymae, (mono búho) se realizó en el IVITAIquitos, Perú con la finalidad de medir parámetros de crecimiento durante el primer semestre de vida en ejemplares nacidos en cautiverio. Se registraron medidas biométricas en 30 neonatos de mono búho desde el nacimiento hasta las 26 semanas de vida con intervalos de 2 semanas. Las medidas biométricas consideradas fueron peso corporal, largo corporal, longitud de brazo, pierna y cola, y perímetro craneano. Se concluyó que las mediadas biométricas registradas están perfectamente correlacionadas con la edad. La ecuación de regresión lineal múltiple $\left(\mathrm{Y}=\mathrm{a}+\mathrm{b}_{1} \mathrm{x}_{1}+\mathrm{b}_{2} \mathrm{x}_{2}+\mathrm{b}_{3} \mathrm{x}_{3}+\mathrm{b}_{4} \mathrm{x}_{4}+\mathrm{b}_{5} \mathrm{x}_{5}+\mathrm{b}_{6} \mathrm{x}_{6}\right)$ nos permite predecir la edad de un Aotus hasta el año de edad a partir de los valores biométricos obtenidos.

Palabras clave: Medidas biométricas, Aotus, cautiverio, parámetros de crecimiento.

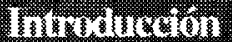

Los primates neotropicales son especialmente valiosos por sus características fisiológicas y bioquímicas análogas a las humanos. Debido a este hecho, los primates han sido objeto desde hace muchos años a

I Práctica privada.

2 Centro de Conservación y Reproducción de Primates No-Humanos (CRCP) - IVITA - IquitosFMV - UNMSM. E.Mail: ivita@tvs.com.pe intensos estudios, contribuyendo en el desarrollo de vacunas contra enfermedades, como la poliomielitis, y ampliando el conocimiento y comprensión de otras enfermedades, como malaria, fiebre amarilla, sarampión, tuberculosis, trastornos mentales y oncogénesis viral, entre otras. (Whitney, 1990; Málaga, 1983; Young et al., 1976).

El Aotus nancymae es la especie que más ventajas ofrece como modelo de estudio biomédico de malaria en el laboratorio, debido a que es susceptible a la infección del 
Plasmodium de origen humano y el curso de la infección es similar al que se registra en el hombre (Young et al., 1976; Rieckmann et al., 1980); por otro lado, también es utilizado en estudios de oftalmología experimental, tanto quirúrgica como terapéutica en la búsqueda de la vacuna contra el Herpes venéreo, genital o tipo II, y en el herpes virus oncogénico (King, 1994).

Para la crianza de esta especie bajo cautiverio se requiere conocer la evolución del crecimiento y desarrollo durante el primer semestre de vida (infantil-juvenil) para establecer una guía de crecimiento normal que permita detectar si las variaciones en peso, talla y otros corresponden a la velocidad de crecimiento esperada.

\section{Materiales y Metodns}

\section{Animales}

El estudio comprendió el seguimiento de 30 crías de Aotus nancymae, provenientes de embarazo de feto único, que durante el período neonatal y postnatal no presentaron anomalías congénitas, diarreas graves ni infecciones. Las crías procedieron de una colonia de 80 parejas reproductoras del Centro de Reproducción y Conservación de Primates (CRCP) de la Estación Experimental IVITA-Iquitos.

\section{Alimentación}

Los animales fueron alimentados a base de una galleta cuyos ingredientes son : harina de soya, harina de trigo, harina de arroz, azúcar, huevos, suplementos vitamínicos y minerales. La galleta aportó en promedio $24,2 \%$ de proteína cruda, $11,8 \%$ de grasa, $4,8 \%$ de fibra cruda. La ración diaria fue de 40 a $50 \mathrm{~g}$ ( 4 a 5 galletas) por individuo adulto y 3 galletas en el juvenil, complementándose con frutos de la región y agua ad libitum.

\section{Recolección de datos}

Se seleccionaron medidas biométricas conocidas, según lo recomendado por la Organización Mundial de la Salud (WHO, 1990) y Gorstein y Sullivan (1994). Dichas medidas fueron peso corporal, largo corporal, longitud de brazo, pierna y cola y perímetro craneano. Los registros de las medidas biométricas se realizaron a las 24 horas de nacidas las crías y cada dos semanas posteriormente hasta completar las 26 semanas de vida. Los datos sobre peso corporal fueron tomados usando balanzas de precisión de 100, 300, 1000 $\mathrm{g}$; una regla metálica graduada en cm para tomar las longitudes de cuerpo y extremidades; y una cinta métrica inextensible y flexible de $30 \mathrm{~cm}$ para registrar el perímetro craneano.

\section{Análisis de datos}

Se determinó la Velocidad Media de Crecimiento (VMC), según Martell et al. (1981). Las medidas tomadas en cada control así como la variación de estas entre dos controles fueron analizadas con el programa estadístico Statgraphics. Se aplicaron las pruebas de correlación lineal múltiple y la prueba de regresión lineal múltiple. para esta última se empleó la ecuación: $Y=$ $a+b_{1} x_{1}+b_{2} x_{2}+b_{3} x_{3}+b_{4} x_{4}+b_{5} x_{5}+b_{6} x_{6}$ en donde $Y$ es la variable dependiente (edad en semanas); a es el intercepto; $b_{1}$, $\mathrm{b}_{2}, \mathrm{~b}_{3}, \mathrm{~b}_{4}, \mathrm{~b}_{5}, \mathrm{y} \mathrm{b}_{6}$, son los coeficientes de regresión; $\mathrm{x}_{1}, \mathrm{x}_{2}, \mathrm{x}_{3}, \mathrm{x}_{4}, \mathrm{x}_{5} \mathrm{y}_{6}$ son las variables independientes (medidas biométricas): Peso, longitud corporal, longitud de cola, longitud de antebrazo, longitud de pierna, perímetro craneano y edad en semanas. 
En el Cuadro 1, se observa los valores del peso corporal. Se comprobó en general que los machos muestran un mayor peso que las hembras desde el nacimiento hasta las 26 semanas de vida. La ganancia de peso cada dos semanas fue de $30.4 \mathrm{~g}$ en promedio. La correlación entre la edad y el peso corporal fue de 0.99 .

El desarrollo de la longitud corporal (Cuadro 2) fue ligeramente mayor en los machos que en las hembras desde el nacimiento. La correlación entre la edad y la longitud corporal fue de 0.98 .

Los Cuadros 3, 4, 5 y 6, muestran el desarrollo de la longitud de la cola, longitud de antebrazo, longitud de pierna y perímetro craneano, respectivamente. En todas estas variables los machos mostraron valores ligeramente mayores que las hembras desde el nacimiento hasta las $26 \mathrm{se}$ manas de vida; no siendo estas diferencias importantes por ser muy pequeñas. Los valores de correlación entre la edad y los parámetros antes indicados fueron 0.97 , $0.99,0.99$ y 0.96 , respectivamente.

En la VMC del peso corporal, desde el nacimiento hasta las 26 semanas de vida, se observa que el incremento de peso promedio por día fue ligeramente mayor en los machos que en las hembras ( $2.37 \mathrm{~g}$ vs $2.17 \mathrm{~g})$.

Con respecto a la VMC de la longitud corporal, durante el periodo estudiado, se observa que la ganancia promedio diaria tanto para machos como para hembras fue de 0.07 $\mathrm{cm}$; la VMC de la longitud de cola, fue ligeramente mayor en machos que en las hembras $(0.12$ vs $0.11 \mathrm{~cm})$; la VMC de la longitud del antebrazo para ambos sexos $(0.2 \mathrm{~cm} /$ día $)$ fue mayor que la longitud de pierna $(0.03 \mathrm{~cm} /$ día); y finalmente en la VMC del perímetro craneano, tanto en los machos como las hembras, presentaron un valor de $0.03 \mathrm{~cm} / \mathrm{d}$.

En el Cuadro 7 se muestran los valores de todas las variables biométricas consideradas en el estudio y su relación con la edad, real y estimada, mediante la ecuación de regresión lineal múltiple anotada anteriormente. Sin duda, la edad esta influenciada por la variación de los seis parámetros estudiados. El modelo utilizado es estadísticamente significativo $(\mathrm{P}<0.0001)$.

\section{Discuston}

Los métodos biométricos utilizados en este estudio para establecer parámetros de crecimiento fueron medidas básicas, que son las recomendadas por la Organización Mundial de la Salud y utilizadas por otros autores (Jones, 1972; Sinclair, 1973; Waterlow et al., 1977).

Los resultados del análisis de correlación múltiple muestran la asociación que existe entre las variales biométricas estudiadas y la edad, la misma que es alta y positiva. Simultaneamente la correlación entre las seis variables biométricas, independientemente de la edad, fue alta y positiva. Esto sugiere que en evaluaciones de crecimiento de primates, como los del presente estudio, la medición de una sola de las variables biométricas podría ser útil para estimar si el desarrollo es adecuado.

Por otro lado, la ecuación de regresión lineal múltiple utilizada nos ha permitido observar la contribución de cada una de las variables en la predicción de la edad. En condiciones de cautiverio esta ecuación permitirá determinar cuales deberían de ser los parámetros biométricos que un individuo debería alcanzar en un determinado momento para así evaluar si el desarrollo del mismo es adecuado (Sokal y Rohlf, 1979). 
Cuadro 1. Evolución del peso corporal (g) de la especie Aotus nancymae desde el nacimiento hasta las 26 semanas de vida.

\begin{tabular}{|c|c|c|c|c|c|c|}
\hline \multirow{2}{*}{ Semana } & \multicolumn{2}{|c|}{$\begin{array}{c}\text { Hembras } \\
(n=16)\end{array}$} & \multicolumn{2}{|c|}{$\begin{array}{c}\text { Machos } \\
(n=14)\end{array}$} & \multicolumn{2}{|c|}{$\begin{array}{l}\text { Promedio General } \\
(n=30)\end{array}$} \\
\hline & Promedio & $\pm \mathrm{IC}^{*}$ & Promedio & $\pm \mathrm{IC}$ & Promedio & $\pm \mathrm{IC}$ \\
\hline 0 & 97.6 & 6.02 & 99.6 & 5.54 & 98.6 & 3.79 \\
\hline 2 & 150.6 & 10.09 & 160.2 & 40.5 & 155.1 & 6.88 \\
\hline 4 & 187.2 & 11.03 & 207.9 & 3.49 & 196.8 & 8.69 \\
\hline 6 & 217.8 & 8.79 & 267.7 & 46.35 & 227.8 & 11.04 \\
\hline 8 & 250.9 & 16.55 & 239.2 & 19.54 & 258.7 & 11.97 \\
\hline 10 & 284.1 & 18.97 & 295.0 & 24.80 & 289.2 & 14.16 \\
\hline 12 & 320.3 & 21.89 & 334.5 & 35.69 & 326.9 & 18.71 \\
\hline 14 & 354.7 & 25.38 & 359.1 & 22.42 & 356.8 & 15.69 \\
\hline 16 & 373.1 & 21.26 & 395.0 & 23.69 & 383.4 & 14.98 \\
\hline 18 & 397.6 & 15.96 & 426.0 & 23.69 & 411.0 & 14.18 \\
\hline 20 & 425.2 & 13.45 & 461.1 & 25.90 & 442.1 & 14.34 \\
\hline 22 & 450.8 & 12.73 & 483.6 & 26.06 & 466.1 & 13.97 \\
\hline 24 & 473.4 & 13.88 & 509.3 & 27.90 & 490.1 & 15.06 \\
\hline 26 & 493.1 & 16.92 & 530.0 & 29.03 & 510.3 & 16.23 \\
\hline
\end{tabular}

* Intervalo de confianza.

Cuadro 2. Evolución de la longitud corporal $(\mathrm{cm})$ de la especie Aotus nancymae desde el nacimiento hasta làs 26 semanas de vida.

\begin{tabular}{|c|c|c|c|c|c|c|}
\hline \multirow{2}{*}{ Semana } & \multicolumn{2}{|c|}{$\begin{array}{l}\text { Hembras } \\
(n=16)\end{array}$} & \multicolumn{2}{|c|}{$\begin{array}{l}\text { Machos } \\
(n=14)\end{array}$} & \multicolumn{2}{|c|}{$\begin{array}{c}\text { Promedio General } \\
(\mathrm{n}=30)\end{array}$} \\
\hline & Promedio & $\pm \mathrm{IC}$ & Promedio & $\pm \mathrm{IC}$ & Promedio & $\pm \mathrm{IC}$ \\
\hline 0 & 12.1 & 0.31 & 12.3 & 0.95 & 12.2 & 0.28 \\
\hline 2 & 13.7 & 0.37 & 14.1 & 1.15 & 13.9 & 0.34 \\
\hline 4 & 15.2 & 0.19 & 15.5 & 0.94 & 15.3 & 0.25 \\
\hline 6 & 16.5 & 0.39 & 16.6 & 0.76 & 16.6 & 0.27 \\
\hline 8 & 17.4 & 0.42 & 17.8 & 0.91 & 17.6 & 0.33 \\
\hline 10 & 18.2 & 0.44 & 18.9 & 0.98 & 18.5 & 0.33 \\
\hline 12 & 19.3 & 0.49 & 20.1 & 1.27 & 19.7 & 0.42 \\
\hline 14 & 20.8 & 0.54 & 21.3 & 1.04 & 21.0 & 0.38 \\
\hline 16 & 21.5 & 0.53 & 21.9 & 0.80 & 21.7 & 0.33 \\
\hline 18 & 22.1 & 0.56 & 22.5 & 0.93 & 22.3 & 0.37 \\
\hline 20 & 22.6 & 0.52 & 23.1 & 1.03 & 22.8 & 0.38 \\
\hline 22 & 23.0 & 0.50 & 23.8 & 1.10 & 23.4 & 0.39 \\
\hline 24 & 23.5 & 0.41 & 24.4 & 0.93 & 24.0 & 0.34 \\
\hline 26 & 24.1 & 0.39 & 25.0 & 1.07 & 24.6 & 0.36 \\
\hline
\end{tabular}


Cuadro 3. Longitud de cola (cm) de la especie Aotus nancymae desde el nacimiento hasta las 26 semanas de vida.

\begin{tabular}{|c|c|c|c|c|c|c|}
\hline \multirow[t]{2}{*}{ Semana } & \multicolumn{2}{|c|}{$\begin{array}{c}\text { Hembras } \\
(n=16)\end{array}$} & \multicolumn{2}{|c|}{$\begin{array}{c}\text { Machos } \\
(n=14)\end{array}$} & \multicolumn{2}{|c|}{$\begin{array}{l}\text { Promedio General } \\
\qquad(\mathrm{n}=30)\end{array}$} \\
\hline & Promedio & $\pm \mathrm{IC}$. & Promedio & $\pm \mathrm{IC}$ & Promedio & $\pm \mathrm{IC}$ \\
\hline 0 & 13.1 & 0.42 & 13.1 & 0.53 & 13.1 & 0.30 \\
\hline 2 & 15.9 & 0.62 & 16.3 & 0.57 & 16.1 & 0.39 \\
\hline 4 & 18.8 & 0.65 & 19.5 & 0.52 & 19.1 & 0.41 \\
\hline 6 & 21.3 & 1.29 & 21.8 & 0.72 & 21.5 & 0.46 \\
\hline 8 & 23.1 & 0.78 & 24.1 & 0.84 & 23.6 & 0.55 \\
\hline 10 & 25.1 & 0.86 & 25.6 & 1.07 & 25.4 & 0.63 \\
\hline 12 & 26.9 & 0.95 & 27.1 & 1.11 & 27.0 & 0.66 \\
\hline 14 & 28.6 & 0.91 & 28.6 & 1.11 & 28.6 & 0.65 \\
\hline 16 & 29.6 & 0.88 & 30.2 & 1.10 & 29.9 & 0.64 \\
\hline 18 & 30.8 & 0.72 & 31.2 & 1.07 & 31.0 & 0.58 \\
\hline 20 & 31.4 & 0.78 & 32.1 & 0.97 & 31.8 & 0.58 \\
\hline 22 & 32.2 & 0.81 & 32.8 & 0.94 & 32.5 & 0.58 \\
\hline 24 & 33.1 & 0.84 & 33.8 & 1.00 & 33.4 & 0.60 \\
\hline 26 & 33.7 & 0.76 & 34.5 & 1.20 & 34.1 & 0.64 \\
\hline
\end{tabular}

Cuadro 4. Longitud de antebrazo $(\mathrm{cm})$ de la especie Aotus nancymae desde el nacimiento hasta las 26 semanas de vida.

\begin{tabular}{|c|c|c|c|c|c|c|}
\hline \multirow[t]{2}{*}{ Semana } & \multicolumn{2}{|c|}{$\begin{array}{l}\text { Hembras } \\
(n=16)\end{array}$} & \multicolumn{2}{|c|}{$\begin{array}{l}\text { Machos } \\
(n=14)\end{array}$} & \multicolumn{2}{|c|}{$\begin{array}{c}\text { Promedio General } \\
(\mathrm{n}=30)\end{array}$} \\
\hline & Promedio & $\pm \mathrm{IC}$ & Promedio & $\pm \mathrm{IC}$ & Promedio & $\pm \mathrm{IC}$ \\
\hline 0 & 3.0 & 0.17 & 3.1 & 0.13 & 3.0 & 0.10 \\
\hline 2 & 3.5 & 0.11 & 3.5 & 0.16 & 3.5 & 0.09 \\
\hline 4 & 4.0 & 0.15 & 3.9 & 0.13 & 3.9 & 0.09 \\
\hline 6 & 4.3 & 0.13 & 4.4 & 0.14 & 4.3 & 0.09 \\
\hline 8 & 4.6 & 0.13 & 4.6 & 0.13 & 4.6 & 0.09 \\
\hline 10 & 4.8 & 0.14 & 4.8 & 0.09 & 4.8 & 0.08 \\
\hline 12 & 5.1 & 0.15 & 5.1 & 0.11 & 5.1 & 0.09 \\
\hline 14 & 5.3 & 0.19 & 5.4 & 0.17 & 5.4 & 0.12 \\
\hline 16 & 5.6 & 0.22 & 5.7 & 0.18 & 5.6 & 0.13 \\
\hline 18 & 5.9 & 0.26 & 5.8 & 0.17 & 5.8 & 0.15 \\
\hline 20 & 6.0 & 0.25 & 6.1 & 0.19 & 6.1 & 0.15 \\
\hline 22 & 6.2 & 0.26 & 6.4 & 0.16 & 6.3 & 0.14 \\
\hline 24 & 6.4 & 0.26 & 6.6 & 0.14 & 6.5 & 0.14 \\
\hline 26 & 6.7 & 0.25 & 6.7 & 0.17 & 6.7 & 0.14 \\
\hline
\end{tabular}


Cuadro 5. Longitud de pierna (cm) de la especie Aotus nancymae desde el nacimiento hasta las 26 semanas de vida.

\begin{tabular}{|c|c|c|c|c|c|c|}
\hline \multirow[t]{2}{*}{ Semana } & \multicolumn{2}{|c|}{$\begin{array}{c}\text { Hembras } \\
(n=16)\end{array}$} & \multicolumn{2}{|c|}{$\begin{array}{l}\text { Machos } \\
(n=14)\end{array}$} & \multicolumn{2}{|c|}{$\begin{array}{l}\text { Promedio General } \\
\qquad(\mathrm{n}=30)\end{array}$} \\
\hline & Promedio & $\pm \mathrm{IC}$ & Promedio & $\pm \mathrm{IC}$ & Promedio & $\pm \mathrm{IC}$ \\
\hline 0 & 3.6 & 0.20 & 3.6 & 0.15 & 3.6 & 0.11 \\
\hline 2 & 4.2 & 0.21 & 4.4 & 0.20 & 4.3 & 0.14 \\
\hline 4 & 4.8 & 0.23 & 5.0 & 0.22 & 4.9 & 0.15 \\
\hline 6 & 5.4 & 0.18 & 5.6 & 0.20 & 5.5 & 0.13 \\
\hline 8 & 5.8 & 0.11 & 6.0 & 0.17 & 5.9 & 0.10 \\
\hline 10 & 6.2 & 0.19 & 6.4 & 0.14 & 6.3 & 0.11 \\
\hline 12 & 6.7 & 0.21 & 6.8 & 0.18 & 6.7 & 0.13 \\
\hline 14 & 7.0 & 0.20 & 7.0 & 0.20 & 7.0 & 0.13 \\
\hline 16 & 7.3 & 0.20 & 7.5 & 0.30 & 7.4 & 0.16 \\
\hline 18 & 7.7 & 0.30 & 7.8 & 0.30 & 7.8 & 0.19 \\
\hline 20 & 8.0 & 0.27 & 8.2 & 0.29 & 8.1 & 0.18 \\
\hline 22 & 8.3 & 0.28 & 8.5 & 0.29 & 8.4 & 0.19 \\
\hline 24 & 8.5 & 0.28 & 8.7 & 0.30 & 8.6 & 0.19 \\
\hline 26 & 8.9 & 0.27 & 9.0 & 0.24 & 8.9 & 0.17 \\
\hline
\end{tabular}

Cuadro 6. Perímetro craneano $(\mathrm{cm})$ de la especie Aotus nancymae desde el nacimiento hasta las 26 semanas de vida.

\begin{tabular}{|c|c|c|c|c|c|c|}
\hline \multirow{2}{*}{ Semana } & \multicolumn{2}{|c|}{$\begin{array}{c}\text { Hembras } \\
(n=16)\end{array}$} & \multicolumn{2}{|c|}{$\begin{array}{l}\text { Machos } \\
(n=14)\end{array}$} & \multicolumn{2}{|c|}{$\begin{array}{l}\text { Promedio general } \\
\qquad(\mathrm{n}=30)\end{array}$} \\
\hline & Promedio & $\pm \mathrm{IC}$ & Promedio & $\pm \mathrm{IC}$ & Promedio & $\pm \mathrm{IC}$ \\
\hline 0 & 11.5 & 0.31 & 11.9 & 0.23 & 11.7 & 0.19 \\
\hline 2 & 12.8 & 0.22 & 12.9 & 0.21 & 12.8 & 0.14 \\
\hline 4 & 13.4 & 0.33 & 13.7 & 0.27 & 13.5 & 0.21 \\
\hline 6 & 13.9 & 0.24 & 14.2 & 0.31 & 14.1 & 0.17 \\
\hline 8 & 14.4 & 0.21 & 14.6 & 0.31 & 14.5 & 0.17 \\
\hline 10 & 14.6 & 0.25 & 14.9 & 0.31 & 14.7 & 0.19 \\
\hline 12 & 15.0 & 0.31 & 15.2 & 0.27 & 15.1 & 0.19 \\
\hline 14 & 15.2 & 0.31 & 15.5 & 0.29 & 15.4 & 0.20 \\
\hline 16 & 15.6 & 0.27 & 15.8 & 0.29 & 15.6 & 0.19 \\
\hline 18 & 15.8 & 0.26 & 15.9 & 0.27 & 15.8 & 0.18 \\
\hline 20 & 15.9 & 0.27 & 16.2 & 0.31 & 16.0 & 0.19 \\
\hline 22 & 16.0 & 0.27 & 16.5 & 0.32 & 16.3 & 0.21 \\
\hline 24 & 16.2 & 0.29 & 16.7 & 0.35 & 16.5 & 0.22 \\
\hline 26 & 16.5 & 0.28 & 16.9 & 0.31 & 16.7 & 0.20 \\
\hline
\end{tabular}


Cuadro 7. Comparación de la edad real y la edad estimada obtenida a partir de la ecuación de regresión lineal múltiple.*

\begin{tabular}{ccccccccc}
\hline Peso & $\begin{array}{c}\text { Longitud } \\
\text { corporal }\end{array}$ & $\begin{array}{c}\text { Longitud } \\
\text { cola }\end{array}$ & $\begin{array}{c}\text { Longitud } \\
\text { antebrazo } \\
\left(\mathrm{X}_{1}\right)\end{array}$ & $\left(\mathrm{X}_{2}\right)$ & $\left(\mathrm{X}_{3}\right)$ & $\begin{array}{c}\text { Longitud } \\
\text { pierna } \\
\left(\mathrm{X}_{4}\right)\end{array}$ & $\begin{array}{c}\text { Perímetro } \\
\left(\mathrm{X}_{5}\right)\end{array}$ & $\begin{array}{c}\text { Edad semanas } \\
\text { craneano }\end{array}$ \\
\cline { 7 - 9 } & $\left(\mathrm{X}_{6}\right)$ & Real & $\begin{array}{c}\text { Esti- } \\
\text { mada }\end{array}$ \\
\hline 98.6 & 12.2 & 13.1 & 3.0 & 3.6 & 11.7 & 24 horas & 0 \\
155.1 & 13.9 & 16.1 & 3.5 & 4.3 & 12.8 & 2 & 2 \\
196.8 & 15.3 & 19.1 & 3.9 & 4.9 & 13.5 & 4 & 5 \\
227.8 & 16.6 & 21.5 & 4.3 & 5.5 & 14.1 & 6 & 7 \\
258.7 & 17.6 & 23.6 & 4.6 & 5.9 & 14.5 & 8 & 9 \\
289.2 & 18.5 & 25.4 & 4.8 & 6.3 & 14.7 & 10 & 11 \\
326.9 & 19.7 & 27.0 & 5.1 & 6.7 & 15.1 & 12 & 13 \\
356.8 & 21.0 & 28.6 & 5.4 & 7.0 & 15.4 & 14 & 15 \\
383.4 & 21.7 & 29.9 & 5.6 & 7.4 & 15.6 & 16 & 17 \\
411.0 & 22.8 & 31.0 & 5.8 & 7.8 & 15.8 & 18 & 20 \\
442.1 & 22.8 & 31.8 & 6.1 & 8.1 & 16.0 & 20 & 22 \\
466.1 & 23.4 & 32.5 & 6.3 & 8.4 & 16.3 & 22 & 24 \\
490.1 & 24.0 & 33.4 & 6.5 & 8.6 & 16.5 & 24 & 25 \\
510.3 & 24.6 & 34.1 & 6.7 & 8.9 & 16.7 & 26 & 28 \\
\hline *Y=4.36 + & $0.01 \mathrm{X}_{1}+0.66 \mathrm{X}_{2}-0.73 \mathrm{X}_{3}+6.04 \mathrm{X}_{4}+4.51 \mathrm{X}_{5}-3.23 \mathrm{X}_{6}$ & & &
\end{tabular}

Los resultados muestran que el mejor parámetro para predecir la edad es el peso, seguido del largo corporal, siendo el perímetro craneano el que menor índice de correlación muestra coincidiendo con lo reportado por Diamond y McDonment, (1993).

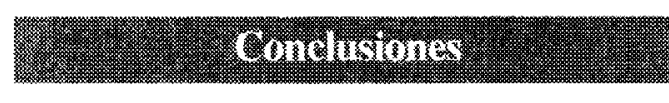

Las medidas biométricas estudiadas están perfectamente correlacionadas con la edad, mostrando un alto grado de asociación positivo ( $r=0.99)$. El peso es la medida biométrica que tiene mayor grado de correlación con la edad. La VMC que se registró cada dos semanas fue de $30.4 \mathrm{~g}$ en hembras y $33.2 \mathrm{~g}$ en machos.

La ecuación de regresión lineal múltiple obtenida para predecir la edad de un Aotus nancymae hasta el año de edad a partir de los seis parámetros biométricos analizados fue la siguiente:

$\begin{aligned} \mathrm{Y}= & 4.36+0.01 \mathrm{x}_{1}+0.66 \mathrm{x}_{2}-0.73 \mathrm{x}_{3}+ \\ & 6.04 \mathrm{x}_{4}+4.51 \mathrm{x}_{5}-3.23 \mathrm{x}_{6}\end{aligned}$ Donde:

$\mathrm{Y}=$ Edad; $\mathrm{X}=$ Peso $\mathrm{X}=$ Longitud corporal; $\mathrm{X}=$ Longitud de cola; $\mathrm{X}=$ =Longitud de antebrazo; $X_{5}^{3}=$ Longitud de pierna; $\mathrm{X}_{6}=$ Perímetro craneano.

\section{Inmtura Cinh}

1. Diamond, I. y J. McDonment. 1993. Collaborative study of birth weight surrogates. Use of a simple anthropometric measurement to predict birth weight. WHO Bulletin. 71(2): 157-163.

2. Gorstein, J. y K. Sullivan. 1994. Issues in the assessment of nutritional status using anthropometry. WHO 
Bulletin. 72(2): 273-283.

3. Jones, C. 1972. Feeding Habits and Body Size. Natural Diets of Wild Primates. En: Pathology of Simian Primates. Part I, pp. 58-77. Ed. R.N.T-WFiennes, London.

4. King, N.W. 1994. The Owl Monkey in Oncogenic Virus Research. En: Aotus: The Owl Monkey. Cap.9, Ed., Janet F.Baer; Richard E.Weller; Ibulaimu Kakoma. Academic Press, Inc.

5. Málaga, C. 1983. Los Primates nohumanos y la investigación biomédica: su importancia en la conservación de las especies. De: "La Primatología en Latinoamérica". Pp: 277-281. Ed., Saavedra, C.; Mittermeier, R.A.; Bastos Santos.

6. Martell, M.; L.A. Bertolini; F. Nieto; S.M. Tenzer; R. Ruggia y R. Belitzki. 1981. Crecimiento y Desarrollo en los dos primeros años de vida posnatal. Organización Panamericana de la Salud, Publicación Científica No 406.

7. Rieckmann, K.; J.E.K. Mrema; P. Marchall y D. Hafner. 1980. Breeding of Aotus Monkey for Human Malaria Research. Bull Pan American Health Organization, 14(3).
8. Sinclair, D. 1973. Human growth after birth. $2^{\mathrm{a}}$ edición. Nueva York: Oxford University Press.

9. Sokal, R. y F.J. Rohlf. 1979. Biometria : Principios y métodos estadísticos en la investigación biológica. pp:534-552. H. Blume Ediciones, Primera edición española.

10. WHO. 1990. Manual de Antropometria Física. WHO Bulletin, pp 2-11.

11. Waterlow, J.C.; R. Buzina; W. Keller $y$ M. Lane. 1977. The presentation and use of height and weight data for comparing the nutritional status of groups of children under the age of 10 years. WHO.55(4) : 489-498.

12. Whitney, R. A. Jr. 1990. Conservación de Primates No Humanos y su Importancia para la Salud Pública. En: "Primates de las Américas". Pp: 205211. Ed., Primo Arámbulo III; Filomeno Encarnación; Jaime Estupiñan; Hugo Samamé; Charles R. Watson; Richard E. Weller. Battelle Seattle Conference Center, Seattle, Washington, USA. Octubre 29-31. OPS/OMS.Battelle Press, Columbus. Richland.

13. Young, M.D.; R. Rossan y D. Baerg. February. 1976. Gorgas Memorial Laboratory, Balboa Heights, Canal Zone. Malaria. Comparative Pathology Bulletin. 8:1. 